\title{
Single particle Raman spectroscopy analysis of the metal-organic framework DUT-8(Ni) switching transition under hydrostatic pressure
}

\author{
Alexander Krylov, ${ }^{\mathrm{a}}$ Irena Senkovska, ${ }^{\mathrm{b}}$ Sebastian Ehrling, ${ }^{\mathrm{b}}$ Mariia Maliuta, ${ }^{\mathrm{b}}$ \\ Svetlana Krylova, ${ }^{\mathrm{a}}$ Evgenia Slyusareva, ${ }^{\mathrm{c}}$ Alexander Vtyurin ${ }^{\mathrm{ac}}$ and Stefan Kaskel $^{\mathrm{b}}$ \\ ${ }^{a}$ Kirensky Institute of Physics, Federal Research Center KSC SB RAS, 660036 Krasnoyarsk, Russia. \\ ${ }^{b}$ Chair of Inorganic Chemistry I, Technische Universitat Dresden, Bergstrasse 66, 01069 Dresden, Germany. \\ c Siberian Federal University, Svobodny Prospect 79, 660041 Krasnoyarsk, Russia
}

Experimental in situ observations of phase coexistence in switch- able metal-organic frameworks are reported to provide a fundamental understanding of dynamic adsorbents that can change their pore structure in response to external stimuli. A prototypical flexible pillared layer framework DUT-8(Ni) (DUT = Dresden University of Technology) was studied under hydrostatic pressure by in situ Raman spectroscopy on single crystals. The closing transition of the open pore phase (op) containing DMF in the pores in silicon oil as a pressure transmitting fluid, as well as the closed pore phase (сp) to op transition under pressure in methanol, were studied. Phase coexistences during both transitions were observed.

Flexible metal-organic frameworks (MOFs) are capable of pronounced structural changes upon the influence of external stimuli, such as presence of gases or liquids, temperature, electromagnetic irradiation or mechanical stress. The dynamic response of highly porous frameworks is a promising feature for applications in gas storage, separation, sensing etc. ${ }^{1 ’ 2}$ However, for establishing flexible MOFs as a widely usable technology platform a fundamental understanding of mechanisms and factors affecting switchability is of paramount importance. Despite very recent research progress in understanding of dynamic structural transformations in porous frameworks supported by DFT, GCMC and hybrid Monte Carlo methods ${ }^{3}$ numerous open questions remain. In particular, the role of surface, particle size, defects, nucleation phenomena, cooperativity etc. are important factors but challenging to delineate both from experimental and theoretical points of view.

In general, established literature distinguishes first-order transitions (discontinuous structural transition) and second- order transitions (continuous transformation) and this categorization may also apply to flexible MOFs as long as only the solid-solid transition is considered. ${ }^{4}$ A first order phase transition is characteristic for many switchable MOFs, showing gating or breathing in adsorption isotherms as evident from the pronounced hysteresis. A prototypical pillared layer $\mathrm{Zn}_{2}(1,4$ - bdc) 2 dabco system (1,4-bdc = terephthalate, dabco - 1,4- diazabicyclo[2.2.2]octane) was recently studied by Keupp and Schmid using Molecular Dynamics simulations under high pressure, postulating a mechanism of first-order phase transition with an interface between the op and cp form, travelling rapidly through the crystallite. ${ }^{5}$

However, the necessity of temporary coexistence of the op and cp phases inside the crystal upon first order phase transition, raises several intriguing questions on the propagation rate of phase transition fronts, the role of cooperativity in this process, appearance and nature of spatially separated regions with distinct structures as well as the question, if the transition can be interrupted, resulting in a coexistence of trapped metastable states and equilibrium phases. The transformation mechanism, the role of cooperativity and phase coexistence questions were previously discussed for MIL-53 (M(OH)(1,4- bdc), $\mathrm{M}=\mathrm{Al}$, Cr, Sc) system, a widely investigated breathable MOF. ${ }^{6-8}$ Recently, Paesani et al. have monitored the breathing of an individual MIL-53(Cr) crystallite upon water adsorption and temperature variation in the environmental TEM showing, that under certain conditions, the diffraction patterns indicated two different lattice structures simultaneously, which might be due to the 
coexistence of the two phases in a single crystal domain. ${ }^{3}$ Rogge et al. ${ }^{10}$ postulated from simulations, that according to the theoretical calculations pillared layer MOF Zn $\mathrm{Z}_{2}(1,4-\mathrm{bdc})_{2}$ dabco (DMOF-1(Zn)) may exhibit metastable cp/op phase coexistence regions under influence of hydrostatic pressure in the pressure range between $0.1 \mathrm{GPa}$ and $0.14 \mathrm{GPa}$. At $0.18 \mathrm{GPa}$, the op state becomes unstable and the material transforms into cp state. Excellent recent reviews summarize the progress in the field of high-pressure studies on MOFs. ${ }^{11,15}$ Mercury intrusion experiments performed on desolvated open pore DMOF$1(\mathrm{Zn} / \mathrm{Cu})$ show the starting transition pressure at 0.051 and $0.193 \mathrm{GPa}$, respectively. ${ }^{16}$ The transition, however, is accompanied by amorphisation after $0.42 \mathrm{GPa}$. In contrast, solvated DMOF-1(Co) shows a first transition to lower symmetry at 0.7 GPa in Daphne oil, and the (pre)amorphous state formation (without breaking of coordination bonds) was detected in the region of 2.19 to $3.46 \mathrm{GPa} .{ }^{17}$ Previous studies revealed, that the mechanical properties as well as threshold characteristics in flexible MOFs are often influenced by the metal in the node and linker functionality. ${ }^{18-24}$

DMOFs, however show only minor response to most guests, and the cell volume change predicted for contraction under pressure is expected to be only $40 \% .{ }^{16}$ We investigated the behaviour of a switchable MOF, namely DUT-8(Ni) $\left(\mathrm{Ni}{ }_{2}(\mathrm{ndc})_{2}(-\right.$ dabco) (ndc - 2,6-naphthalene dicarboxylate)), ${ }^{25}$ capable of reversible phase transitions accompanied by a giant change of porosity and crystallite volume (up to $240 \%$ ). ${ }^{26}$ It transforms into a closed pore phase (сp) upon desolvation and can be reversibly transformed into the open pore phase (op) by adsorption of selected fluids (Fig. 1a and b). ${ }^{25,26}$

For DUT-8(Ni) the cp-op structural transformation upon adsorption of many fluids shows a characteristic discontinuous transformation. It is accompanied by a large hysteresis, typical for a first-order transition. ${ }^{25}$ During in situ gas adsorption/X-ray diffraction experiments performed on powdered DUT-8(Ni)_cp samples the simultaneous presence of the op and cp phases is always observed. ${ }^{26}$

This coexistence was interpreted as crystallites switching consecutively after each other. The gate opening is an activated process and crystals varying in size, morphology, defect concentration, surface barriers, etc. are expected to show different gate opening pressures due to differences in the activation barriers.

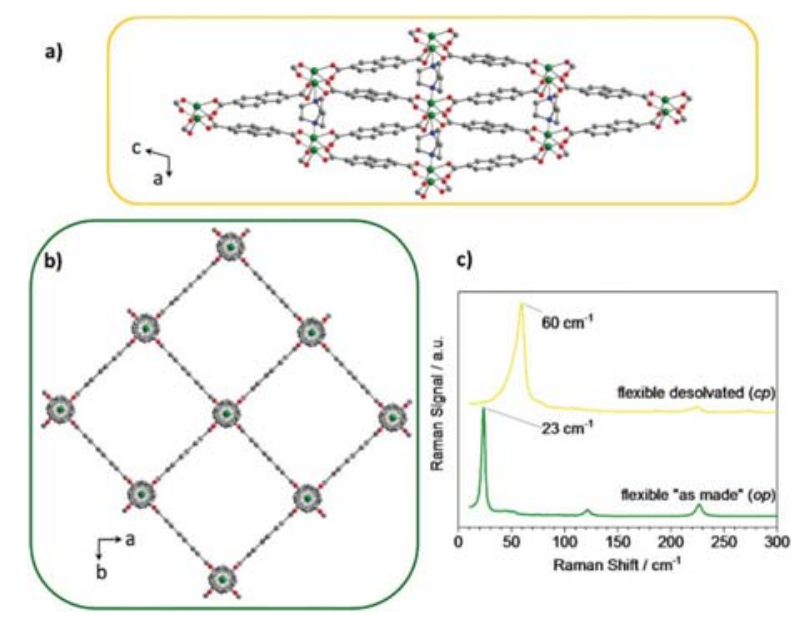

Fig. 1 (a) Crystal structure of DUT-8(Ni)_cp (CCDC 1034317);

(b) crystal structure of DUT-8(Ni)_op (CCDC 760964) (the solvent molecules are omitted).

(c) Raman spectra of DUT-8(Ni)_op (green) and DUT-8(Ni)_cp (yellow) at atmospheric conditions. ${ }^{32}$

The dependence of flexible behaviour from crystal size has been demonstrated for DUT-8 and other MOFs. ${ }^{27-31}$

An alternative explanation for the appearance of $\mathrm{cp}$ and op phases is the coexistence in single crystals as recently postulated. ${ }^{10}$ Both hypotheses (coexistence in one single crystal vs. separated crystals switching consecutively) are not mutually exclusive but have important implications for rational tuning of switchable adsorbents and their applications. 
In the following we analyse the switching mechanism of DUT-8(Ni) in order to detect supposed coexistence of op and cp phase in single crystals under hydrostatic conditions using Raman spectroscopy, since this technique can discriminate between op and cp phases in the low-frequency Raman region $\left(<200 \mathrm{~cm}^{-1}\right)$ unambiguously, as reported previously (Fig. 1c). ${ }^{22,32}$ Moreover, it can be applied for structure monitoring in situ, and can be easily performed on single crystals, opening the possibility to address specific questions and rule out the effects associated with the particle size distribution in a powder. ${ }^{17,32-34}$ Raman spectroscopy is also useful in case diffraction quality of single crystals suffers due to fracturing, ${ }^{35}$ as amorphisation and bond breaking can be clearly detected. ${ }^{17,36-38}$

The closing behaviour of DUT-8(Ni)_op was investigated on a single crystal, containing N,N-dimethylformamide (DMF) in the pores and silicon oil as pressure transmittance medium. At a minimum pressure of 0.02 GPa applied in the experiment, only one signal at $23 \mathrm{~cm}^{-1}$ is present, corresponding to the pure op state of the structure. However, a minimal increase of pressure up to $0.05 \mathrm{GPa}$ leads to the appearance of an additional line at $60 \mathrm{~cm}^{-1}$ and, correspondingly, to the beginning of the closing process (Fig. 2 and Fig. S5, ESIf). The equilibration time after pressure jump was at least 30 min, ensuring proper equilibration.

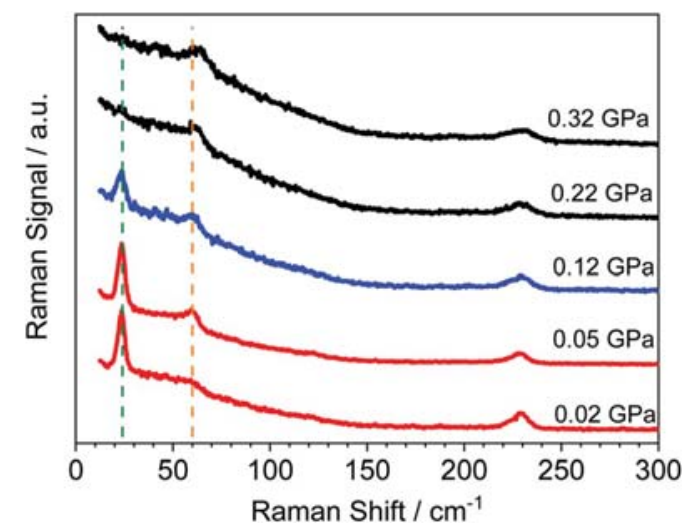

Fig. 2 Raman spectra of DUT-8(Ni)_op under hydrostatic pressure in silicone oil (region up to 300 cm_1, for full spectrum see Fig. S5, ESI†). The position of the characteristic op and cp signals are marked as green and orange dashed lines, respectively.

Interestingly, both lines, at $23 \mathrm{~cm}^{-1}$ (characteristic for the op phase) and $60 \mathrm{~cm}^{-1}$ (characteristic to the cp phase) appear in the spectra, indicating the presence of cp phase and op phases simultaneously within the same crystal. This observation suggests a coexistence of the op and cp phase as it has been observed during in situ gas adsorption/X-ray diffraction experiments ${ }^{26}$ and might indicate that cp and op domains in DUT-8(Ni) are able to coexist inside one single crystal.

In solvated DUT-8(Ni) crystal under hydrostatic pressure, both phases coexist up to $0.12 \mathrm{GPa}$, however the ratio between the cp to op domains changes with pressure, as evident from the changes in the peak area ratio of the characteristic signals at 23 and $60 \mathrm{~cm}^{-1}$, respectively (Fig. 3). Taking into consideration that the measurement spot (ca. $2 \mathrm{pm}$ in diameter or $c a .8 \mathrm{pm}{ }^{3}$ in volume) is much smaller, than the single crystal size (up to $70 \mathrm{pm}$ in length, Fig. S2, ESIf), one can suppose a wider distribution of op and cp domains through the whole crystal. However, the formation of grain boundaries or partially disconnected domains in a particular crystal habit that still appears visually as an intact macrocrystal of characteristic shape cannot be fully ruled out at this point (Fig. S3, ESIf). At $0.32 \mathrm{GPa}$, the line at $23 \mathrm{~cm}^{-1}$ completely disappears in the spectrum and we assume that the complete sample is transformed into the closed pore phase state. 


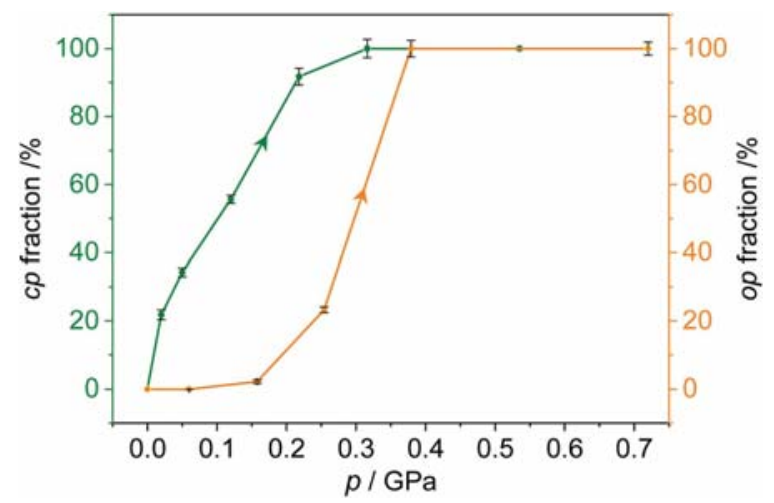

Fig. 3 Presence of the corresponding phase fraction (for details see ESIf) in the single crystal depending on the pressure. The green symbols represent the experiment performed on the open framework (left axis), the yellow symbols represent the experiment performed on cp phase in methanol (right axis).

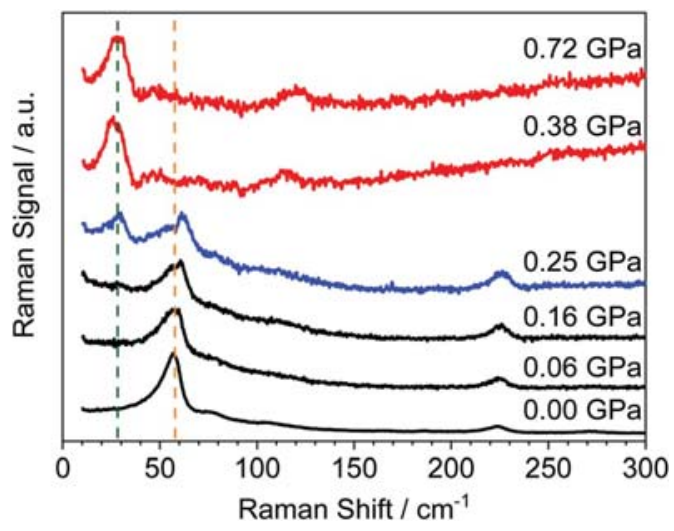

Fig. 4 Raman spectra of DUT-8(Ni)_cp under hydrostatic pressure in methanol (region up to $300 \mathrm{~cm}^{-1}$, for full spectrum see Fig. S6, ESIf). The position of the characteristic op and cp signals are marked as green and orange dashed lines, respectively.

To investigate the opening mechanism of DUT-8(Ni)_cp, the pressure transmitting medium was changed to methanol. From our previous liquid phase adsorption experiments it is known, that liquid alcohols, including methanol, are not able to provoke structural opening of DUT-8(Ni)_cp at room temperature and atmospheric pressure, although the kinetic diameters of the molecules are smaller than the pore opening of the framework (for $\mathrm{MeOH} 3.8 \mathrm{~A}$ ). ${ }^{39}$ Therefore, methanol preserves the closed pore state at ambient pressure and may potentially penetrate into the MOF at higher pressure to form the op phase, making it an ideal pressure-transducing medium for the in situ studies. Indeed, after the crystal of DUT-8(Ni)_cp was placed in to the diamond anvil cell in methanol, below 0.06 GPa, the signal characteristic for the cp phase only is present in the measured spectra (Fig. 4 and Fig. S6, ESIf). However, compression to 0.16 GPa leads to the appearance of the characteristic op phase line at $23 \mathrm{~cm}^{-1}$, while the line of the cp phase at $60 \mathrm{~cm}^{-1}$ is still dominant. At $0.25 \mathrm{GPa}$, the intensity of the op phase signal increases, and coexistence of both characteristic lines in the spectrum indicate again the coexistence of open and closed pore phases in the same macro-crystal, similar to the observation made by the compression of the DUT-8(Ni)_op solvated with DMF (Fig. 2). This experiment is comparable to the gas phase adsorption process along the metastable regime supporting the hypothesis of phase coexistence in one crystal.

The cp phase line at $60 \mathrm{~cm}^{-1}$ disappears completely at the pressure of $0.38 \mathrm{GPa}$, where the sample completely transforms into the op phase. Presumably, the "hydrostatic gate opening pressure"' can be associated with the energetic barrier for the structure opening, similar to the "'gas gate opening pressure' in the gas adsorption experiments. In the case of methanol, the increased fluid pressure compensates the dispersion stabilization ${ }^{40}$ leading to the phase transition to the op phase, which is not possible at atmospheric pressure.

Even though the solvates were different in both experiments, one can conclude that the closing process of the solvated op phase requires less mechanical pressure than the opening of the cp phase, representing the different activation barriers for the opening and closing processes in agreement with fluid adsorption experiments. However, each process is irreversible in the same experimental run, meaning that the opening of the cp phase cannot be achieved by the pressure release only. To open the ср phase, the presence of interpenetrating fluid, such as methanol, able to stabilize the op phase after opening is indispensable.

Summarising, Raman spectroscopy in situ investigations on DUT-8(Ni) under hydrostatic pressure provide meaningful insights into the phase transformation mechanism of dynamic MOFs, due to the distinct differences in the characteristic lattice vibration frequencies of the op $\left(23 \mathrm{~cm}^{-1}\right)$ and $\mathrm{cp}\left(60 \mathrm{~cm}^{-1}\right)$ phases. The op phase with DMF in the pores starts to close at 0.05 
GPa. The cp phase can be opened in methanol at $0.16 \mathrm{GPa}$. No continuous drift of lines from $23 \mathrm{~cm}^{-1}$ (op) to $60 \mathrm{~cm}^{-1}$ (cp) and vice versa was found, and only continuous redistribution of characteristic line intensities occurs. Hence, no intermediate lattice structure present and the transformation between closed and open phases under pressure occurs as first order transition, similar to the observations made by in situ adsorption/powder diffraction experiments during gas adsorption.

More importantly, the in situ experiments show, that in some pressure ranges characteristic lines corresponding to the open and closed pore phases coexist simultaneously in the Raman spectrum of a crystal, indicating the coexistence of cp and op domains, arrested in a metastable state.

This work is financially supported by the Russian Foundation for Basic Research No. 18-02-00754 and German research foundation (FOR 2433, MOF-switches). The authors would like to thank Dr V. Bon for support and scientific discussions.

\section{Conflicts of interest}

There are no conflicts to declare.

\section{Notes and references}

1 Z. Chang, D. Yang, J. Xu, T.-L. Hu and X.-H. Bu, Adv. Mater., 2015, 27, 5432-5441.

2 G. Maurin, C. Serre, A. Cooper and G. Ferey, Chem. Soc. Rev., 2017, 46, 3104-3107.

3 J. H. Lee, S. Jeoung, Y. G. Chung and H. R. Moon, Coord. Chem. Rev., 2019, 389, 161-188.

4 J. B. Clarke, J. W. Hastie, L. H. E. Kihlborg, R. Metselaar and M. Thackeray, Pure Appl. Chem., 1994, 66, 577.

5 J. Keupp and R. Schmid, Adv. Theory Simul., 2019, 2, 1900117.

6 A. Boutin, F.-X. Coudert, M.-A. Springuel-Huet, A. V. Neimark, G. Ferey and A. H. Fuchs, J. Phys. Chem. C, 2010, 114, 22237-22244.

7 F. Salles, H. Jobic, A. Ghoufi, P. L. Llewellyn, C. Serre, S. Bourrelly, G. Ferey and G. Maurin, Angew. Chem., Int. Ed., 2009, 48, 8335-8339.

8 M.-A. Springuel-Huet, A. Nossov, Z. Adem, F. Guenneau, C. Volkringer, T. Loiseau, G. Ferey and A. Gedeon, J. Am. Chem. Soc., 2010, 132, 11599-11607.

9 L. R. Parent, C. H. Pham, J. P. Patterson, M. S. Denny, S. M. Cohen, C. Gianneschi and F. Paesani, J. Am. Chem. Soc., 2017, 139, 13973-13976.

10 S. M. J. Rogge, M. Waroquier and V. Van Speybroeck, Nat. Commun., 2019, 10, 4842.

11 I. E. Collings and A. L. Goodwin, J. Appl. Phys., 2019, 126, 181101.

12 F.-X. Coudert, Chem. Mater., 2015, 27, 1905-1916.

13 A. Clearfield, Dalton Trans., 2016, 45, 4100-4112.

14 S. C. McKellar and S. A. Moggach, Acta Crystallogr., Sect. B: Struct. Sci., Cryst. Eng. Mater., 2015, 71, 587-607.

15 V. Bon, E. Brunner, A. Poppl and S. Kaskel, Adv. Funct. Mater., 2020, 1907847, DOI: 10.1002/adfm.201907847.

16 J. Wieme, S. M. J. Rogge, P. G. Yot, L. Vanduyfhuys, S.-K. Lee, J.- S. Chang, M. Waroquier, G. Maurin and V. Van Speybroeck, J. Mater. Chem. A, 2019, 7, 22663-22674.

17 M. Andrzejewski, N. Casati and A. Katrusiak, Dalton Trans., 2017, 46, 14795-14803.

18 L. Vanduyfhuys, S. Rogge, J. Wieme, S. Vandenbrande, G. Maurin, M. Waroquier and V. Speybroeck, Nat. Commun., 2018, 9, 204.

19 S. Henke, W. Li and A. K. Cheetham, Chem. Sci., 2014, 5, 2392-2397.

20 J.-P. Zhang, H.-L. Zhou, D.-D. Zhou, P.-Q. Liao and X.-M. Chen, Natl. Sci. Rev., 2017, 5, 907-919.

21 N. Klein, H. C. Hoffmann, A. Cadiau, J. Getzschmann, M. R. Lohe, S. Paasch, T. Heydenreich, K. Adil, I. Senkovska, E. Brunner and S. Kaskel, J. Mater. Chem., 2012, 22, 10303-10312. 
22 S. Ehrling, I. Senkovska, V. Bon, J. D. Evans, P. Petkov, Y. Krupskaya, V. Kataev, T. Wulf, A. Krylov, A. Vtyurin, S. Krylova, S. Adichtchev, E. Slyusareva, M. S. Weiss, B. Buochner, T. Heine and S. Kaskel, J. Mater. Chem. A, 2019, 7, 21459-21475.

23 A. S. Munn, R. S. Pillai, S. Biswas, N. Stock, G. Maurin and R. I. Walton, Dalton Trans., 2016, 45, 4162-4168.

24 A. Schneemann, P. Vervoorts, I. Hante, M. Tu, S. Wannapaiboon, Sternemann, M. Paulus, D. C. F. Wieland, S. Henke and A. Fischer, Chem. Mater., 2018, 30, 1667-1676.

25 N. Klein, C. Herzog, M. Sabo, I. Senkovska, J. Getzschmann, Paasch, M. R. Lohe, E. Brunner and S. Kaskel, Phys. Chem. Chem. Phys., 2010, 12, 11778-11784.

26 V. Bon, N. Klein, I. Senkovska, A. Heerwig, J. Getzschmann, Wallacher, I. Zizak, M. Brzhezinskaya, U. Mueller and S. Kaskel, Phys. Chem. Chem. Phys., 2015, 17, 17471-17479.

27 C. Zhang, J. A. Gee, D. S. Sholl and R. P. Lively, J. Phys. Chem. C, 2014, 118, 20727-20733.

28 S. Krause, V. Bon, H. Du, R. E. Dunin-Borkowski, U. Stoeck, I. Senkovska and S. Kaskel, Beilstein J. Nanotechnol., 2019, 10, 1737-1744.

29 S. Krause, V. Bon, I. Senkovska, D. Toobbens, D. Wallacher, S. Pillai, G. Maurin and S. Kaskel, Nat. Commun., 2018, 9, 1573.

30 Y. Sakata, S. Furukawa, M. Kondo, K. Hirai, N. Horike, Y. Takashima, H. Uehara, N. Louvain, M. Meilikhov, T. Tsuruoka, Isoda, W. Kosaka, O. Sakata and S. Kitagawa, Science, 2013, 339, 193-196.

31 M. Shivanna, Q.-Y. Yang, A. Bajpai, S. Sen, N. Hosono, S. Kusaka, Pham, K. A. Forrest, B. Space, S. Kitagawa and M. J. Zaworotko, Sci. Adv., 2018, 4, eaaq1636.

32 A. Krylov, A. Vtyurin, P. Petkov, I. Senkovska, M. Maliuta, V. Bon, T. Heine, S. Kaskel and E. Slyusareva, Phys. Chem. Chem. Phys., 2017, 19, 32099-32104.

33 S. Sakaida, K. Otsubo, O. Sakata, C. Song, A. Fujiwara, M. Takata and H. Kitagawa, Nat. Chem., 2016, 8, $377-383$.

34 G. Kumari, N. R. Patil, V. S. Bhadram, R. Haldar, S. Bonakala, T. K. Maji and C. Narayana, J. Raman Spectrosc., 2016, 47, 149-155.

35 S. 0ien-0degaard, G. C. Shearer, D. S. Wragg and K. P. Lillerud, Chem. Soc. Rev., 2017, 46, 4867-4876.

36 N. Ovsyuk and S. Goryainov, Appl. Phys. Lett., 2006, 89, 134103.

37 S. K. Deb, M. A. Rekha, A. P. Roy, V. Vijayakumar, S. Meenakshi and B. K. Godwal, Phys. Rev. B: Condens. Matter Mater. Phys., 1993, 47, 11491-11494.

38 H. Mao, J. Xu, Y. Hu, Y. Huang and Y. Song, J. Mater. Chem. A, 2015, 3, 11976-11984.

39 N. Kavoosi, T. Savchenko, I. Senkovska, M. Maliuta, V. Bon, A. Eychmuller and S. Kaskel, Microporous Mesoporous Mater., 2018, 271, 169-174.

40 J. Wieme, K. Lejaeghere, G. Kresse and V. Van Speybroeck, Nat. Commun., 2018, 9, 4899. 